\title{
Patients' descriptions of angina symptoms: a qualitative study of primary care patients
}

\author{
Melvyn M Jones, Claire Somerville, Gene Feder and Gill Foster
}

\author{
ABSTRACT \\ Background \\ Initial diagnosis of angina in primary care is based on \\ the history of symptoms as described by the patient in \\ consultation with their GP. Deciphering and \\ categorising often complex symptom narratives, \\ therefore, represents an ongoing challenge in the early \\ diagnosis of angina in primary care. \\ Aim \\ To explore how patients with a pre-existing angina \\ diagnosis describe their symptoms.

\section{Method} \\ Semi-structured interviews were conducted with 64 \\ males and females, identified from general practice \\ records as having received a diagnosis of angina within \\ the previous 5 years.

\section{Results} \\ While some patients described their angina symptoms \\ in narratives consistent with typical anginal symptoms, \\ others offered more complex descriptions of their \\ angina experiences, which were less easy to classify. \\ The latter was particularly the case for severe coronary \\ artery disease, where some patients tended to \\ downplay chest pain or attribute their experience to \\ other causes.

\section{Conclusion} \\ Patients with a known diagnosis of angina do not \\ always describe their symptoms in a way that is \\ consistent with Diamond and Forrester's diagnostic \\ framework for typicality of angina. Early diagnosis of \\ angina in primary care requires that GPs operate with a \\ broad level of awareness of the various ways in which \\ their patients describe their symptoms.

\section{Keywords} \\ angina pectoris; coronary artery disease; diagnosis; \\ qualitative research; primary health care.
}

\section{INTRODUCTION}

This article examines angina as a diagnosis of chest pain due to coronary artery disease (CAD). Although angina is strictly a symptom, this diagnosis is made exclusively by interpreting the patient's history of chest pain. There is little role for physical examination, and most useful tests for angina can only be accessed by GPs through referral, once a provisional diagnosis is made. It is known that key elements of a patient's history of chest pain - the typicality of these symptoms ${ }^{2}$ (Box 1 ) and other risk factors - predict the likelihood of CAD. ${ }^{3}$ Reliance on interpreting a well-described set of symptoms has been called the angina 'canon' (Box 2), ${ }^{4}$ and its use is observed in secondary care settings. GPs, however, seem to use a more loose set of rules to diagnose underlying CAD. ${ }^{5}$ Adherence to such a canon of typicality may result in a significant proportion of patients with underlying $C A D$ being missed $\left(30 \%\right.$ in one study $\left.{ }^{6}\right)$, leading to underrecognition of $\mathrm{CAD},{ }^{7}$ and, perhaps, partially explaining avoidable CAD-related deaths..$^{8-11}$

The diagnosis of angina is a complex clinical interaction, with the patient describing their pain or symptoms and the clinician recognising the

MM Jones, MD, MSc, MRCGP, UCL Medical School, Research Department of Primary Care and Population Health, University College London, and Warden Lodge, Cheshunt. C Somerville, BA, MA, PhD; G Foster, RGN, BA, MSc, Barts and The London School of Medicine and Dentistry, Queen Mary University of London, Institute for Health Sciences Education, London. G Feder, MD, FRCGP, Unit of Academic Primary Health Care, University of Bristol, Bristol.

Address for correspondence

Dr Melvyn Jones, UCL Medical School, Research Department of Primary Care and Population Health, Royal Free Campus, London, NW3 2PF.

E-mail: melvyn.jones@ucl.ac.uk

Submitted: 2 October 2009; Editor's response: 2 December 2009; final acceptance: 16 June 2010.

(c)British Journal of General Practice 2010; 60: 735-741.

DOI: 10.3399/bjgp10X532378 


\section{How this fits in}

Rigid adherence to Diamond and Forrester's canon of anginal typicality may lead to some under-recognition of coronary artery disease, presenting as chest pain. Clinicians need to be aware that patients with coronary artery disease will use non-canonical terms to describe their symptoms. Some symptoms may need to be added to the canon, such as breathlessness. Care may be needed in excluding angina on the basis of sharp pain, and clinicians should be aware that patients who have had myocardial infarction may underplay subsequent symptoms of angina.

\section{Box 1. Diamond and Forrester's classification of chest pain} in relation to angina diagnosis. ${ }^{2}$

- Typical (definite angina):

- substernal discomfort with characteristic quality and duration,

- provoked by exertion or emotional stress,

- relieved by rest or short-acting nitrate drug.

The characteristic quality of the pain in stable angina is dull, heavy, or aching (not sharp or burning), and its duration should be minutes (not seconds or hours).

- Atypical (probable angina) meets two of the above criteria.

- Non-cardiac chest pain meets one or none of the above criteria.

\section{Box 2. What is the angina canon?}

In the performance (elucidation) of 'cardiac' and 'non-cardiac' chest pain, consultations consist of posing and repetition of a limited set of questions, so that doctors can structure the patient narrative. The dialogue includes the quality and duration of the pain, and precipitating and relieving factors, all corresponding to Diamond and Forrester's features of chest pain. ${ }^{2}$ It is the continued use of this framework, over a prolonged period of time, that gives it canonical status (adapted from Somerville et $\left.a /{ }^{4}\right)$.

\section{Box 3. Topic guide.}

The patient is asked to talk about their symptoms and the nature of their chest pain. The specific prompts to the interviewee were then:

- 'Have you had any pain or discomfort in your chest, jaw, or neck in the last year?'

followed by:

- 'Please describe, in your own words, the symptoms you experience'. characteristics of the pain in such a way that they are led to think of angina. ${ }^{12}$ Yet, describing the sensation of pain is difficult, ${ }^{13}$ particularly in the case of chest pain. As cardiac symptoms are often not perceived as pain, ${ }^{14-16}$ this makes doctors' questions about chest pain more problematic. Adding to the complexity, different groups report their chest symptoms using different descriptions; examples include the role of sex ${ }^{15,17,18}$ and ethnicity. ${ }^{19-21}$ Additionally, as chest pain is common and CAD is a relatively rare cause of chest pain found in primary care,,$^{22-24}$ there is again further potential for missed diagnoses.

Previous research has prospectively followed up patients identified (by epidemiological surveys) as having chest pain, ${ }^{3,25}$ or followed people presenting to their GPs with chest pain. ${ }^{24}$ This approach potentially misses people with a wider range of angina-related symptoms. The current study started by examining people diagnosed as having CAD which had been identified by several methods (including after an acute cardiac event), and then identified their symptoms retrospectively.

This study sought to explore how people with established angina describe their symptoms, and whether the process of telling clinicians about symptoms made patients any more likely to use terms from the angina canon.

\section{METHOD}

\section{Setting}

Patients with suspected and confirmed angina were recruited from two general practices in 2005, and seen in a study clinic based at a London hospital and in a practice on the border between suburban Essex and North London. The qualitative investigation examined a subset of a larger epidemiological study. ${ }^{26}$

The sample population was recruited by searching two general practice patient registers to identify males and females, actively registered and aged over 40 years, with one or more of the following:

- Read Code for angina pectoris diagnosis (G33) in the past 5 years;

- Code for nitrates (BNF code 2.6.1) in the past 5 years;

- Read Code for ischaemic heart disease (G3) in the past 5 years;

- Prescribed glyceryl trinitrate (GTN) in the past 18 months;

- Read Code for chest pain (R065z) or chest pain text search in the past 18 months; and/or

- Cardiologist referral in the past 5 years.

Lists of potentially eligible participants were screened by the GPs in the practices to exclude those with terminal illness or severe mental illness, 
or those that the GP thought it would be inappropriate to contact according to their own criteria. Potential participants were subsequently invited to study clinics. ${ }^{26}$

Interviews were conducted by three nurses, two GPs, and one public health physician, and audio recorded. The interview was semi-structured according to a topic guide (see Box 3) and did not focus on Diamond and Forrester's diagnostic framework. ${ }^{2}$

Interviews were transcribed verbatim, and methods derived from grounded theory were used to analyse the data. ${ }^{27}$ Transcripts were read and initially analysed separately by two researchers. Data were coded to produce lists of substantive themes and emergent categories which were then compared and discussed. These coded data were grouped under broader categories and compared against original transcripts for consistency and contextual verification. Categories were compared against one another to eliminate overlap and inconsistencies, and also to ensure that no new themes were revealed. The authors discussed the coded data and agreed on salient themes.

To augment the qualitative analysis with diagnostic data, records and transcripts were examined for indicators of coronary disease, such as the GP's diagnostic label (a record of angina or other CAD diagnoses) and medication (a record of nitrate use).

\section{RESULTS}

A pragmatic subsample of 64 patients from the full study were interviewed: data are presented from 48 of these patients with stronger supportive evidence of CAD. ${ }^{26}$

The majority of interviewees who gave their ethnicity were white British (Table 1), but other ethnic groups were oversampled, including south Asian, Chinese, and African Caribbean. One interview was conducted via a family translator, with the remainder carried out in English. Of those interviewed, the majority showed supportive evidence of CAD (reports of previous myocardial infarction [MI], $n=19$; angiography or coronary surgery, $n=17$; investigations such as exercise stress tests, $n=10$; or isotope/thallium scans, $n=$ $2)$; however, a minority $(n=16)$ had weaker evidence of underlying CAD, such as clinician diagnosis alone or just a prescription of GTN. There was evidence of other cardiovascular disease, such as aortic aneurysm or stroke, and some evidence of other heart disease, such as arrhythmia or heart failure. Interviews lasted between 3 and 25 minutes (mean 5.8 minutes). Saturation was achieved as no new themes emerged from the transcripts.

\section{Themes}

Data clustered around three canonical-related categories and a total of nine themes and subthemes, as listed below (Boxes 1 and 2).

- Symptoms that are recognisable to a clinician as 'canonical' angina:

- exertional component to pain symptoms,

- impact of medication,

- duration of symptoms.

- Non-'canonical' angina symptoms (not part of the canon but commonly communicated by patients as a symptom of angina, such as symptom severity):

- breathlessness,

- attribution of symptoms.

- Ambiguous symptoms possibly related to angina (but central to the patient experience of angina; for example, 'feels like indigestion'):

- vague descriptions,

- patients' euphemisms,

- difficulty describing symptoms,

- underplaying of symptoms.

\section{Canonical symptoms}

Exertional component to pain symptoms. The data suggest many patients describe a group of symptoms that are recognisable to a clinician as angina and fit the canon, involving a tightening or pressure across the chest, and these symptoms are related to exertion and a duration of less than 10 minutes:

'No it don't affect me chest at all. Only if I start walking fast then, there's then I get angina. Then I slow down and it's alright ... when I get the pain I leave it a while and a while, I let it go for about 5 or 6, 10 minutes. If it ain't gone then I take the spray [GTN].' (White British male, 64 years; previous $\mathrm{Ml} /$ cardiac arrest)

A mixture of terms were used by responders to describe the pain, but 'tight', or 'tightening' were by far the most commonly used (over half of those who used such terms). Nevertheless, a range of other phrases were used, including synonyms of tight such

\section{Table 1. Comparison of interview} subsample with main study.

\begin{tabular}{lcc} 
Characteristic & Interview sample & Main study ${ }^{26}$ \\
\hline Sample size, $n$ & 64 & 510 \\
\hline Mean age, years (range) & $66.5(45-77)$ & $58.4(42-89)$ \\
\hline Sex, \% male & 40 & 56 \\
\hline Ethnicity, \% white British & 76 & 96 \\
\hline
\end{tabular}


as 'pressure' and 'constriction'. Non-canonical terms such as 'sharp' and its synonym 'stabbing' were also used quite frequently (about a quarter of those who used adjectives). More vague terms were also commonly used, including 'dull', 'discomfort', 'ache', 'tingling', 'niggly', and 'stitchy'.

Impact of medication. The impact of medication, particularly the quick relief associated with GTN, helped patients (and their doctors) to clarify the meaning of their symptoms:

'That [GTN] seems to clear it you know. Within about 10 minutes I'm alright.' (Female on GTN, 75 years; exercise electrocardiogram [ECG] and coronary artery bypass graft [CABG], two vessels, uses GTN)

\section{Non-canonical symptoms}

Breathlessness. Breathlessness was regularly reported by two-thirds of this sample, and it is a symptom that is increasingly recognised as a component of angina. ${ }^{28}$

'Ah yes I get breathe er I get caught for breath ... I have to take a tablet under the tongue [GTN] when I get that.' (White Irish male, 67 years; CABG in 2000, still uses GTN)

Attribution of symptoms. A recurring theme was patients' attribution of symptoms that are highly likely to have an ischaemic cause, to other causes, particularly with respect to indigestion. Other problems, such as respiratory causes like emphysema, were also blamed. More prosaic explanations were also offered, such as old age or a lack of fitness:

'Er it feels like I could have indigestion but I'm not sure. So I take a tablet underneath my tongue just in case.' (Male, 75 years; exercise ECG and CABG, two vessels, uses GTN).

There was an important confusion or uncertainty for some patients (and their doctors) ${ }^{29}$ as to the cause of breathlessness from either angina or comorbidity, such as chronic obstructive pulmonary disease. The problem may be confounded by possible misunderstanding about the role of 'puffers' (inhalers, such as salbutamol) or 'pumps' (sprays like GTN), with a similar lay meaning as symptom relievers:

'So maybe some of the pain that I put down for asthma is that, and I don't know ... sometime you do get a bit of tightening ... and I take the pump and it relieves it and I think oh well ...' (Female, 52 years; two previous Mls, uses GTN)

\section{Ambiguous symptoms}

Vague descriptions. Many patients described symptoms that were not suggestive of angina, yet the participants felt they were important and related to the condition:

'It just started off like er a stabbing pain ... er it's a funny pain, it's er not one that you ignore it feels like urgency, you've gotta do something about it you can't ignore it.' (Black British female, 53 years; using GTN, previous MI, exercise ECG, no angiogram, doctor diagnosis of angina, and had a thallium scan)

Patients linked these symptoms to the concept of angina not being perceived as a 'pain':

'I never had a chest pain, no, nothing. I had this discomfort.' (Female, 77 years; two previous Mls, angiography and CABG)

Patients' euphemisms. Participants used a mixture of similes, synonyms, and slang to describe their symptoms:

'... it's like as if all the stuffin's been knocked out of me.' (White British male, 47 years; previous MI and an angiogram in 1988 and a doctor diagnosis of angina, regular GTN)

'... erm hard in your chest.' (Female, 75 years; exercise ECG and coronary bypass surgery of two vessels, uses GTN)

Difficulty describing symptoms. A striking theme was the difficulty that some patients had in describing their symptoms of chest pain. Some responders were terse, almost monosyllabic in their descriptions, while others struggled to describe their pain. There was often recurring hesitation (long pauses of 'umms' and 'hmms') when starting to describe symptoms, which is only partly captured in transcripts:

\begin{abstract}
'Um ... a feeling of tightness in the middle of the chest um ... particularly um when walking and I need to stop and rest and that helps ... um I can recall I had the feeling of er sort of breathlessness.' (Male, 87 years; uses GTN underwent an exercise test)
\end{abstract}

Underplaying of symptoms. The underplaying of symptoms was evident in several interviews, particularly where there was discordance between 
symptoms and perceptions of severity:

'Silly geezer. Silly old git. Other than that [reference to bypass and medical catastrophes], I'm alright. I'm fit.' (Male, 64 years; previous MI, cardiac arrest, and severe rest angina)

'Well I might find a little pain ... Well I did use the spray er practically every morning er when I take the dog out for a walk.' (White British male, 62 years; previous MI)

This finding fits with work suggesting denial occurs where males in coronary care units perceive their pain as 'probably transient and likely to go away'. ${ }^{18}$

Patients who had experienced previous $\mathrm{Ml}$ also tended to underplay subsequent anginal symptoms, as they were less dramatic and seemed to be perceived as less important:

'Ah not so much as pain but is tightness of chest.' (Chinese male, 59 years; angina and MI, had angiogram [two-vessel disease] and angioplasty and stent, on GTN)

'Well l've never had the crushing sensation or anything like that. Yeah if l've done something strenuous ... or walked very fast all, out of breath and [puffs] and want to rest.' (White British male, 64 years; angina 1991, abnormal angiogram 1997 and bypass, Ml and cardiac arrest)

\section{DISCUSSION}

\section{Summary of main findings}

The main findings of this study are that even among those patients with proven CAD, many do not use 'accepted' CAD narratives to describe their anginal symptoms, and instead maintain their own unique symptom descriptions. That this group has noncanonical symptoms may not surprise GPs. However, we would have expected people who have been through this system of GP referral, hospital outpatients, and even coronary surgery to have become, to some extent, schooled by clinicians in the in the terminology of the anginal canon.

A proportion of responders in this study group had symptoms that do not fit the angina canon, despite these patients having proven CAD, and this adds credence to suggestions that Diamond and Forrester's typicality, ${ }^{2}$ as a diagnostic test with known prognostic characteristics, ${ }^{1}$ may have limitations in that it may be missing a proportion of patients (possibly as much as one-third) with symptomatic CAD. ${ }^{6}$

Why is it that some of these people with CAD do not fit the canon? There are several possible explanations. Their symptoms may be anginal, but the canon may be too rigid for them to be included, thereby raising the biggest safety concerns for typicality as a diagnostic test for CAD. They may have CAD, but could be describing symptoms from other comorbidities, such as reflux. Or, once they have had their chest pain validated as CAD, could these patients be more willing to accept other noncardiac symptoms as anginal? ${ }^{30}$ Nevertheless, the canon built on Diamond and Forrester's work cannot be discarded, as patients with atypical chest pain are recognised to have an intermediate risk of underlying CAD between non-cardiac chest pain and typical chest pain. The canon is still vital and it provides the best evidence-based test available for diagnosing angina.

To understand this discordance between patient and doctor, we need to look at the language used, as it is essential in describing angina symptoms. Some patients in this study had a much better command of language, using more terms or other linguistic tools, such as similes, to describe their symptoms, while others struggled or provided very sparse descriptions. Variation in linguistic ability may help to explain suboptimal recognition, treatment, and referral by doctors ${ }^{29,31}$ among groups such as ethnic minorities ${ }^{21,32}$ where language may not be shared with their clinician.

Underplaying of symptoms is also observed among some of these participants; particularly among males. ${ }^{18}$ This may be important in those people with a previous $\mathrm{Ml}$ who appear to be assuming that unless their pain is as severe as their $\mathrm{MI}$, it is not important. Such groups are still at considerable risk of suffering subsequent cardiac events. ${ }^{25}$

\section{Strengths and limitations of the study}

The findings of this study suggest a possible explanation for late or under-diagnosis of CAD presenting as chest pain in primary care, in that even patients with a confirmed diagnosis of coronary disease often describe their chest pain in terms that are not consistent with a clinical diagnosis of angina. Some of these patients may, of course, have chest pain of non-cardiac origin, but that is unlikely to account for all the patients presenting with symptoms outside the diagnostic canon.

The study recruited a broad range of people with possible anginal symptoms from within primary care, which enabled minimisation of the selection bias which is often observed in such studies undertaken in secondary care. Saturation of themes was achieved, but the investigation has also been able to focus on those who were known to have established CAD. Those with weaker evidence of 
CAD present generally similar symptoms, but it is less certain that their symptoms originate from CAD.

While it is known that patients presented in this study show evidence of CAD, it cannot be determined whether it is in fact ischaemic myocardium that is causing their chest pain. It has not been possible to follow up these patients to ascertain the prognostic implications of these symptoms, yet even with follow-up, considerable methodological difficulties would arise. The fact that only audio recording was used in interviews also has limitations, as it was not possible to study non-verbal communication which can be important. ${ }^{33}$ Finally, sampling identified a subgroup of patients who possibly do not have heart disease, and some interviews that were too short to generate sufficient data.

\section{Comparison with existing literature}

This paper extends and complements previous work by two of the authors on performance of the angina canon, examining how chest pain symptoms were shaped into a diagnosis in a hospital clinic. ${ }^{4}$ The current study is different in three ways. First, discordant views were explored, and their correspondence with the 'angina canon' was examined. Second, the study occurred at a different point in the care pathway for angina; that is, in primary care after the diagnosis has been established. Finally, the work was methodologically different in that interviews were taped rather than recorded in field notes, which allowed more detailed capture of the precise terms that patients used.

This study suggests that some symptoms may need to be added to the canon, such as breathlessness, as their inclusion in the angina symptomatology is reinforced in other literature, ${ }^{28,34}$ and described as an 'angina equivalent'. ${ }^{35}$

The findings in this paper are consistent with Scarry's description of 'pain defying language'. ${ }^{13}$ Pain is also not well remembered ${ }^{36}$ so participants may poorly recall their symptoms when they eventually get seen by the GP, or in clinic, although there is some suggestion that chronic or recurrent pain is more easily recalled. ${ }^{36}$

White and Johnson's work relates to the area of symptom interpretation. ${ }^{18}$ The meaning of symptoms is important for the participants in this study; after all, chest pain carries the 'inherent threat of death'. ${ }^{37}$ It may be that the process of symptom description ('What is it I am feeling?') is hindered by a search for meaning or denial ('Does this pain mean I am heading towards a heart attack?').

\section{Implications for future research}

Patients who describe symptoms that readily fit the diagnostic canon, who describe the 'right' sort of pain, are likely to undergo further investigation for CAD. However, for those whose experience and descriptions of chest pain deviate from the canon, the pathway to the diagnosis of underlying CAD may be delayed or missed altogether. Further work on the inclusion of a broader set of non-canonical symptoms of chest pain as a trigger for investigation of $C A D$, is needed to determine whether the current criteria for initial diagnosis of angina are adequately capturing the CAD population.

To examine prognosis epidemiologically in a population over time, complex patient narratives need to be reduced to a simple summary of symptoms, yet that process may itself lose key prognostic information. There is a need to develop better ways of capturing patients' actual descriptions and to match these to morbidity and mortality outcomes. Use of routine GP databases may help, but GPs act as interpreters of what has been said and what is subsequently transcribed.

Further work may also include the prognostic significance of sharp pain, used by clinicians as a test of exclusion for angina ('negative predictors'4), and the findings of this work would suggest that this process needs careful attention.

\section{Funding body}

Melvyn Jones would like to thank the British Heart Foundation for the provision of funds to assist in data collection (BHF Junior Research Fellowship FS/03/011/15132) and the Department of Health for funding of the pilot study: 'The appropriateness of coronary revascularisation: new insights from the ACRE study and pilot work for a new prospective study in primary care'.

\section{Ethics committee}

This study was approved by Trent MREC, as part of a pilot for an angina prognosis study and to search GP records. Consent was sought from participants, to record and use quotes.

\section{Competing interests}

The authors have stated that there are none.

\section{Acknowledgement}

Professor Harry Hemingway, UCL.

\section{Discuss this article}

Contribute and read comments about this article on the Discussion Forum: http://www.rcgp.org.uk/bjgp-discuss

\section{REFERENCES}

1. Chun AA, McGee SR. Bedside diagnosis of coronary artery disease: a systematic review. Am J Med 2004; 117(5): 334-343.

2. Diamond GA, Forrester JS. Analysis of probability as an aid in the clinical diagnosis of coronary-artery disease. N Engl J Med 1979; 300(24): 1350-1358

3. Croft PR, Thomas E. Chest pain and subsequent consultation for coronary heart disease: a prospective cohort study. $\mathrm{Br} \mathrm{J} \mathrm{Gen} \mathrm{Pract}$ 2007; 57(534): 40-44.

4. Somerville $\mathrm{C}$, Featherstone $\mathrm{K}$, Hemingway $\mathrm{H}$, et al. Performing stable angina pectoris: an ethnographic study. Soc Sci Med 2008; 66(7): 1497-1508.

5. Hani MA, Keller H, Vandenesch J, et al. Different from what the textbooks say: how GPs diagnose coronary heart disease. Fam Pract 2007; 24(6): 622-627.

6. Sekhri N, Feder G, Junghans $\mathrm{C}$, et al. How effective are rapid access 
chest pain clinics? Prognosis of incident angina and non-cardiac chest pain in 8762 consecutive patients. Heart 2007; 93(4): 458-463.

7. Hemingway H, Shipley M, Britton A, et al. Prognosis of angina with and without a diagnosis: 11 year follow up in the Whitehall II prospective cohort study. BMJ 2003; 327(7420): 895.

8. Kannel WB, Doyle JT, McNamara PM, et al. Precursors of sudden coronary death. Factors related to the incidence of sudden death. Circulation 1975; 51(4): 606-613.

9. Alonzo AA, Simon AB, Feinleib M. Prodromata of myocardial infarction and sudden death. Circulation 1975; 52(6): 1056-1062.

10. Kuller LE, Lilienfield AB, Fisher RU. Epidemiological study of sudden and unexpected deaths due to arteriosclerotic heart disease. Circulation 1966; 34(6): 1056-1068.

11. Owen-Smith V, Hannaford PC, Elliott AM. Increased mortality among women with Rose angina who have not presented with ischaemic heart disease. Br J Gen Pract 2003; 53(495): 784-789.

12. Ruston A, Clayton J, Calnan M, Green J. Patients' action during their cardiac event: qualitative study exploring differences and modifiable factors. BMJ 1998; 316(7137): 1060-1065.

13. Scarry E. The body in pain. New York: Oxford University Press, 1985.

14. Hofgren C, Karlson BW, Gaston Johansson F, Herlitz J. Word descriptors in suspected acute myocardial infarction: a comparison between patients with and without confirmed myocardial infarction. Heart Lung 1994; 23(5): 397-403.

15. Albarran JW, Clarke BA, Crawford J. 'It was not chest pain really, can't explain it!' An exploratory study on the nature of symptom experienced by women during their myocardial infarction. J Clin Nurs 2007; 16(7): 1292-1301.

16. Miller CL. Symptom reflections of women with cardiac disease and advanced practice nurses: a descriptive study. Prog Cardiovasc Nurs 2003; 18(2): 69-76.

17. Philpott S, Boynton PM, Feder G, Hemingway H. Gender differences in descriptions of angina symptoms and health problems immediately prior to angiography: the ACRE study. Soc Sci Med 2001; 52(10): 1565-1575

18. White AK, Johnson M. Men making sense of their chest pain niggles, doubts and denials. J Clin Nurs 2000; 9(4): 534-541.

19. Johnson PA, Lee TH, Cook EF, et al. Effect of race on the presentation and management of patients with acute chest pain. Ann Intern Med 1993; 118(8): 593-601.

20. Chaturvedi N. Ethnic differences in cardiovascular disease. Heart 2003; 89(6): 681-686

21. Fischbacher CM, Bhopal R, Unwin N, et al. The performance of the Rose angina questionnaire in South Asian and European origin populations: a comparative study in Newcastle, UK. Int J Epidemiol
2001; 30(5): 1009-1016.

22. Svavarsdóttir AE, Jónasson MR, Gudmundsson GH, Fjeldsted K. Chest pain in family practice. Diagnosis and long-term outcome in a community setting. Can Fam Physician 1996; 42: 1122-1128.

23. Ruigomez A, Rodriguez LA, Wallander MA, et al. Chest pain in general practice: incidence, comorbidity and mortality. Fam Pract 2006; 23(2): 167-174.

24. Nilsson S, Scheike M, Karlsson LG, et al. Chest pain and ischaemic heart disease in primary care. Br J Gen Pract 2003; 53(490): 378-382.

25. Lampe FC, Whincup PH, Wanamethee SG, et al. Chest pain on questionnaire and prediction of major ischaemic heart disease events in men. Eur Heart J 1998; 19(1): 63-73.

26. Junghans $C$, Feder G, Hemingway H, et al. Recruiting patients to medical research: double blind randomised trial of 'opt-in' versus 'opt-out' strategies. BMJ 2005; 331(7522): 940.

27. Glaser BG, Strauss A. The discovery of grounded theory: strategies for qualitative research. Chicago IL: Aldine Publishing Co, 1967.

28. Christopher Jones R, Pothier CE, et al. Prognostic importance of presenting symptoms in patients undergoing exercise testing for evaluation of known or suspected coronary disease. Am J Med 2004; 117(6): 380-389.

29. Gardner K, Chapple A. Barriers to referral in patients with angina: qualitative study. BMJ 1999; 319(418): 421.

30. Thompson DR, Lewin RJP. Coronary disease: management of the post-myocardial infarction patient: rehabilitation and cardiac neurosis. Heart 2000; 84(1): 101-105.

31. Richards HM, Reid ME, Watt GCM. Socioeconomic variations in responses to chest pain: qualitative study. $B M J 2002$; 324(7349): 1308.

32. Feder G, Crook AM, Magee P, et al. Ethnic differences in invasive management of coronary disease: prospective cohort study of patients undergoing angiography. BMJ 2002; 324(7336): 511-516.

33. Marcus GM, Cohen J, Varosy PD. The utility of gestures in patients with chest discomfort. Am J Med 2007; 120(1): 83-89.

34. Cook DG, Shaper AG. Breathlessness, angina pectoris and coronary artery disease. Am J Cardiol 1989; 63(12): 921-924.

35. Braunwald E, Zipes DP, Libby P. Heart disease: a textbook of cardiovascular medicine. 6th edn. Philadelphia: WB Saunders Co, 2001.

36. Erskine A, Morley S, Pearce S. Memory for pain: a review. Pain 1990; 41(3): 255-265.

37. Fitzsimons D, Parahoo K, Stringer M. Waiting for coronary artery bypass surgery: a qualitative analysis. J Adv Nurs 2000; 32(5): $1243-1252$. 